\title{
Clinical Presentation and Imaging Findings of Patients with Dural Arteriovenous Fistulas with an Angiographic Pseudophlebitic Pattern
}

\author{
(D)W. Brinjikji, (D).J. Cloft, and (D) G. Lanzino
}

\begin{abstract}
BACKGROUND AND PURPOSE: The pseudophlebitic pattern is an increasingly recognized angiographic manifestation of chronic venous congestion in the setting of a cranial dural arteriovenous fistula. We sought to study the clinical and radiologic manifestations of patients with the pseudophlebitic pattern.
\end{abstract}

\begin{abstract}
MATERIALS AND METHODS: We retrospectively reviewed a cohort of patients with dural arteriovenous fistulas evaluated at our institution from 2008 to 2020. Angiograms were reviewed to classify dural arteriovenous fistulas and document the presence or absence of a pseudophlebitic pattern, defined as the presence of serpiginous and tortuous collateral, bridging, and cortical veins with an associated delay in circulation time of the normal brain. We then studied the association between the pseudophlebitic pattern and clinical presentation and MR imaging findings.
\end{abstract}

RESULTS: Two hundred one patients were included. Patients with a pseudophlebitic pattern had more hemorrhage $(22.8 \%$ versus $8.4 \%, P=.005)$, gait changes and ataxia $(6.0 \%$ versus $0.0 \%, P=0.002)$, cognitive changes $(6.9 \%$ versus $1.4 \%, P=.04)$, and seizures (8.6\% versus $2.1 \%, P=.03$ ). On MR imaging, the pseudophlebitic pattern was associated with higher rates of cerebral edema $(70.9 \%$ versus $2.9 \%, P<.0001)$, chronic hemosiderin deposition and microhemorrhage (17.3\% versus $2.2 \%, P=.0002)$, and dilated transmedullary veins $(47.1 \%$ versus $0.0 \%, P<.0001)$. When we considered only patients with malignant fistulas, there was no difference in hemorrhage at presentation between the 2 groups $(22.6 \%$ versus $22.8 \%, P=.99)$. Patients with a pseudophlebitic pattern did have higher rates of nonhemorrhagic neurologic deficits $(24.1 \%$ versus $9.4 \%, P=.03)$.

CONCLUSIONS: The pseudophlebitic pattern was associated with high rates of brain parenchymal changes and neurologic symptoms in this cohort of patients with dural arteriovenous fistulas.

ABBREVIATION: $\mathrm{dAVF}=$ dural arteriovenous fistula

$t$ is well-known that the primary determinant of the natural history of a cranial dural arteriovenous fistula (dAVF) is the pattern of venous drainage as lesions that drain directly into leptomeningeal cortical veins have an annual risk of nonhemorrhagic deficit of $7 \%$ and an annual risk of intracranial hemorrhage of $8 \%$ compared with rates of close to $0 \%$ for lesions with anterograde venous drainage into the dural venous sinuses. ${ }^{1}$ One factor that has not been examined as thoroughly as the presence of retrograde leptomeningeal venous drainage is the presence of a pseudophlebitic pattern on conventional angiography. ${ }^{2-4}$ A pseudophlebitic pattern was first described in the setting of dAVFs by

Received June 15, 2020; accepted after revision August 5.

From the Departments of Radiology and Neurosurgery, Mayo Clinic, Rochester, Minnesota.

Please address correspondence to Waleed Brinjikji, MD, Mayo Clinic, Department of Radiology, 200 lst St SW, Rochester, MN 55905; e-mail:

Brinjikji.waleed@mayo.edu; @wbrinjikji

http://dx.doi.org/10.3174/ajnr.A6811
Willinsky et $\mathrm{al}^{4}$ to characterize the tortuous and engorged venous veins identified on the venous phase of the brain circulation (ie, not during the venous phase of the dAVF itself). These dilated serpiginous veins are the result of chronic and severe venous congestion and have been associated with high rates of nonhemorrhagic neurologic deficits as well as hemorrhage itself.

To better understand the significance of the pseudophlebitic pattern on conventional angiography, we performed a retrospective review of our institutional series of dAVFs. The aim of this study was to characterize the brain MR imaging findings, demographics, and clinical presentation of patients with dAVFs who have a pseudophlebitic pattern on conventional angiography.

\section{MATERIALS AND METHODS \\ Patient Population}

Following institutional review board approval, we identified all patients with cranial dAVFs seen at our institution from 2008 to 
2020 as part of a prospectively maintained data base. All included patients had a conventional cerebral angiogram for evaluation of their dural arteriovenous fistula and underwent clinical evaluation by a neurologist and neurosurgeon. Patients who did not consent to the use of their medical records for research were excluded from this study. Patients who did not undergo conventional angiography examining the bilateral internal carotid arteries and posterior circulation were excluded.

\section{Data Collection}

All charts were reviewed to collect information on demographics (age and sex) and clinical presentation. For clinical presentation, we made note of the initial symptoms that the patient was manifesting at the time of diagnosis of the dural arteriovenous fistula. Clinical symptoms were categorized as the following: 1) hemorrhage, 2) incidental, 3) bruit/tinnitus, 4) ocular or visual, and 5) myelopathic symptoms, and 6) ataxia, 7) headache, 8) hydrocephalus, 9) seizures, and 10) other (including dementia and other cognitive defects).

\section{Review of Angiograms}

Angiograms were reviewed by an endovascular neurosurgeon and interventional neuroradiologist. We collected the following data on angioarchitecture: 1) Borden classification, 2) Cognard classification, 3) fistula location, 4) presence of venous dilation, 5) presence of a venous varix, 6) presence of the pseudophlebitic pattern, and 7) draining vein stenosis. The presence of the pseudophlebitic pattern was based on the analysis of the venous drainage of the cerebellar or cerebral hemispheres and was defined by the presence of serpiginous and tortuous collateral, bridging, and cortical veins with an associated delay in circulation time. Venous dilation was defined as greater dilation of the cerebral veins than usual. Venous varix was defined as a venous dilation with a diameter more than twice as large as that of the veins proximal and distal to the varix. Fistula location was categorized as cavernous sinus, transverse sigmoid sinus, tentorial, anterior cranial fossa, middle cranial fossa, foramen magnum, hypoglossal/ jugular foramen, convexity, superior sagittal sinus, extensive, and multiple. ${ }^{4,5}$ Tentorial dural arteriovenous fistulas were categorized according to a previously defined classification system. ${ }^{6}$

\section{Cross-Sectional Imaging Review}

All patients with a brain MR imaging underwent review by a diagnostic and interventional neuroradiologist. In general, brain MRIs included T2, T2/FLAIR, sagittal T1, axial T1 postcontrast, and $\mathrm{T} 2{ }^{\star}$-weighted imaging (either gradient recalled-echo or SWI) and were performed at $1.5 \mathrm{~T}$ or $3 \mathrm{~T}$. Images were reviewed for the presence of the following: 1) cerebral edema, 2) chronic hemosiderin or microhemorrhage on $\mathrm{T}^{*}$ images, 3 ) venous infarction, 4) dilated transmedullary veins on SWI or postcontrast T1-weighted imaging, 5) sinus thrombosis, and 6) cortical venous reflux on 3D-TOF-MRA. All patients underwent imaging on a 1.5 or $3 \mathrm{~T}$ scanner. A variety of scanners were used due to the long timeframe of this study.

\section{Statistical Analysis}

Patients were divided into pseudophlebitic and nonpseudophlebitic groups. Baseline characteristics, clinical presentation, and angioarchitectural and cross-sectional imaging findings were compared across groups. A $\chi^{2}$ test was used for categoric variables, and a Student $t$ test was used to compare continuous variables. All statistical analyses were performed using JMP 14.0 (SAS Institute).

\section{RESULTS}

\section{Patient Population}

A total of 201 patients were included in this study. A pseudophlebitic pattern was seen in 58 patients $(28.9 \%)$, and a no pseudophlebitic pattern was seen in 143 patients $(71.1 \%)$. The mean patient age was $59.1 \pm 13.7$ years; 104 patients $(51.7 \%)$ were men and 97 patients (48.3\%) were women. The 3 most common presenting symptoms were tinnitus/bruit (86 patients, $42.8 \%$ ), visual/ocular symptoms (37 patients, 18.4\%), and hemorrhage (25 patients, $12.5 \%)$. These data are summarized in Table 1. Case examples are shown in Figs 1-3.
Table 1: Demographics and clinical presentation

\begin{tabular}{lcccc}
\hline & Overall & Nonpseudophlebitic & Pseudophlebitic & $\boldsymbol{P}$ \\
\hline No. & 201 & $143(71.1)$ & $58(28.9)$ & - \\
Mean age (SD (yr) & $59.1(13.7)$ & $60.5(13.5)$ & $58.4(13.8)$ & .32 \\
Sex & & & & \\
Male (No.) (\%) & $104(51.7)$ & $64(44.8)$ & $40(69.0)$ & \\
Female (No.) (\%) & $97(48.3)$ & $79(55.2)$ & $18(31.0)$ & .002 \\
Clinical presentation & & & & \\
$\quad$ (No.) (\%) & & & & .005 \\
Hemorrhage & $25(12.5)$ & $12(8.4)$ & $13(22.8)$ & .24 \\
Incidental & $22(11.0)$ & $18(12.6)$ & $4(6.9)$ & .51 \\
Headache & $23(11.4)$ & $15(10.5)$ & $8(13.8)$ & .28 \\
Visual/ocular symptoms & $37(18.4)$ & $29(20.3)$ & $8(13.8)$ & .0001 \\
Tinnitus/bruit & $86(42.8)$ & $77(53.9)$ & $9(15.5)$ & .04 \\
Cognitive changes & $6(3.0)$ & $2(1.4)$ & $4(6.9)$ & .002 \\
Gait changes/ataxia & $4(2.0)$ & $0(0.0)$ & $4(6.0)$ & .11 \\
Hydrocephalus & $1(0.5)$ & $0(0.0)$ & $1(1.7)$ & .03 \\
Seizure & $8(4.0)$ & $3(2.1)$ & $5(8.6)$ & .12 \\
Myelopathy & $7(3.5)$ & $3(2.1)$ & $4(6.0)$ & .0001 \\
Other & $17(8.5)$ & $6(4.2)$ & $11(18.9)$ & \\
\hline
\end{tabular}

Note:-SD indicates standard deviation; yr, year; No., number; \%, percentage.

\section{Baseline Characteristics}

Overall, there was no difference in mean age in the nonpseudophlebitic and pseudophlebitic groups $(P=.32)$. There was a higher proportion of men in the pseudophlebitic group than in the nonpseudophlebitic group $(69.0 \%$ versus $44.8 \%, P=.002)$. Regarding clinical presentation, patients with a pseudophlebitic pattern were more likely to present with hemorrhage (22.8\% versus $8.4 \%, P=.005)$, gait changes and ataxia (6.0\% versus $0.0 \%$, $P=0.002)$, cognitive changes $(6.9 \%$ versus $1.4 \%, P=.04)$, and seizure (8.6\% versus $2.1 \%, P=.03$ ). Patients in the nonpseudophlebitic group were more likely to present with tinnitus/ bruit (53.9\% versus $15.5 \%, P<.0001$ ). 


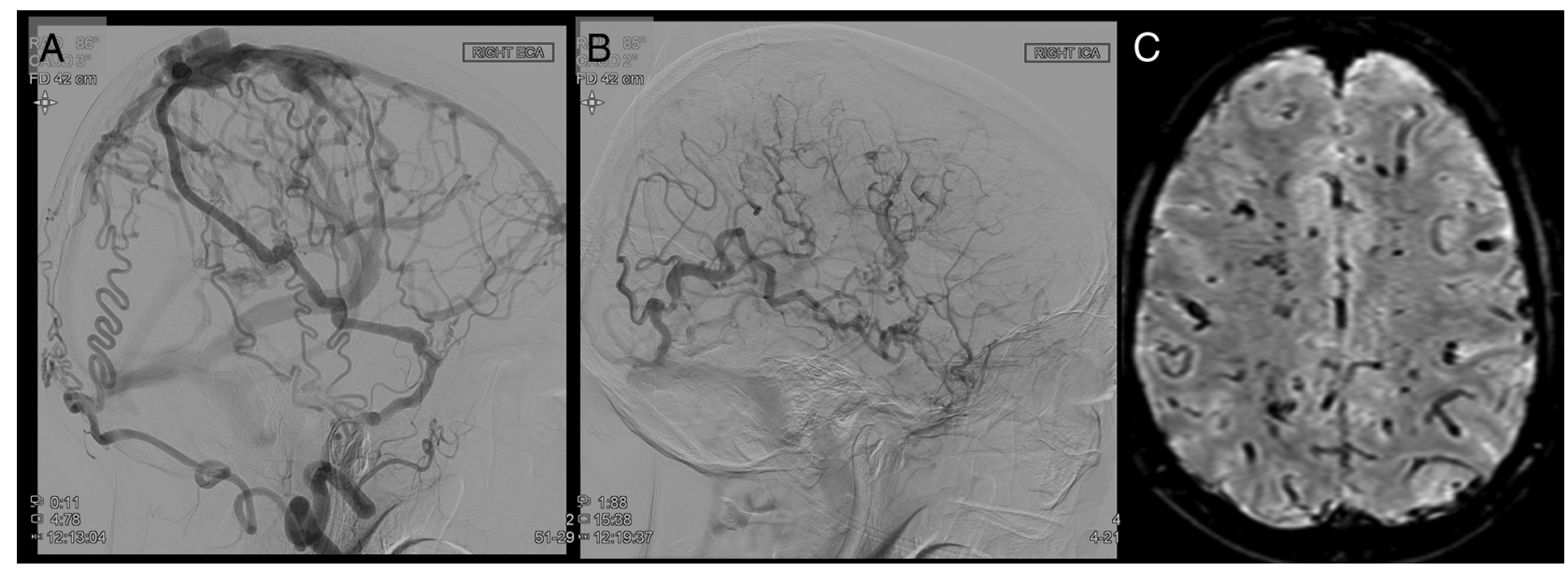

FIG 1. Pseudophlebitic pattern in a superior sagittal sinus fistula in a patient presenting with voice hoarseness. A, Right external carotid artery (ECA) injection shows the superior sagittal sinus fistula with reflux into the cortical veins. B, Right ICA cerebral angiogram shows dilated corkscrew veins in the right cerebral hemisphere during the parenchymal venous phase. There is stagnation of contrast due to the high venous pressure. C, SWI shows dilated T2-hypointense transmedullary veins reflective of venous hypertension.

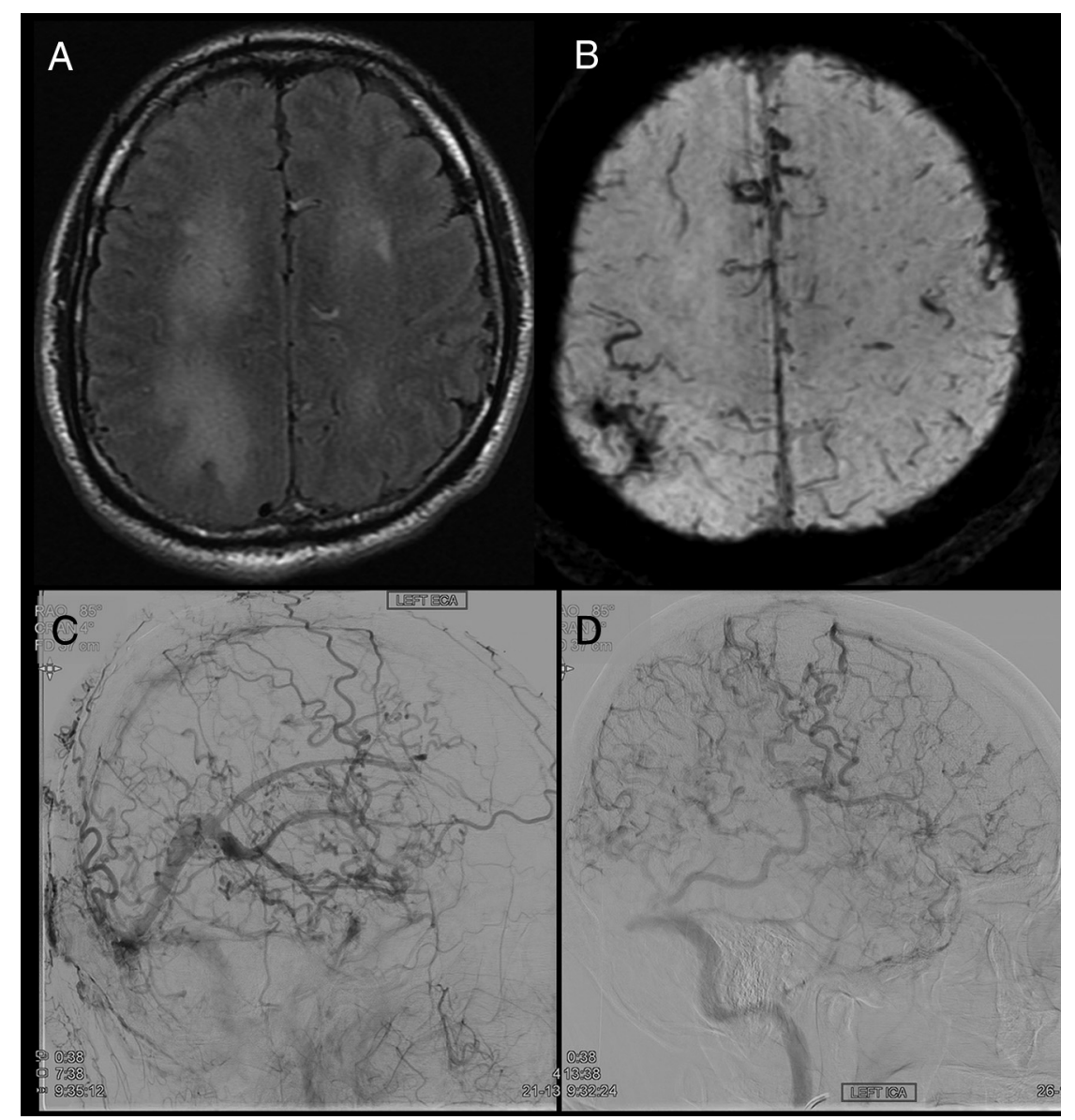

FIG 2. A pseudophlebitic pattern in a patient with an extensive dural arteriovenous fistula presenting with cognitive decline. A, FLAIR imaging shows parenchymal FLAIR signal in the bilateral cerebral hemispheres, right greater than left. $B$, SWI shows a chronic hemorrhagic in the right parietal lobe and some dilated transmedullary veins in the left hemisphere. $C$, Left external carotid artery (ECA) injection shows the superior sagittal sinus and torcular fistulas with reflux into the deep venous system and parenchymal veins. $D$, Left ICA injection shows dilated corkscrew parenchymal cerebral veins, which have some stagnation consistent with a pseudophlelebitic pattern.

\section{Angiographic Findings and dA VF Characteristics}

There was a significant difference in the location of fistulas between groups $(P<.0001)$. Patients with a nonpseudophlebitic pattern were more likely to have fistulas in the transverse-sigmoid sinuses $(40.6 \%$ versus $8.6 \%, P<.0001)$ and cavernous sinus $(16.1 \%$ versus $6.9 \%, P<.0001)$. Meanwhile, patients with a pseudophlebitic pattern were more likely to have the fistulas located in the tentorium $(51.7 \%$ versus $16.8 \%$, $P<.0001)$ and superior sagittal sinus (8.6\% versus $0.7 \%, P=.003$ ).

Patients with a pseudophlebitic pattern were also more likely to have malignant fistulas with Borden II/III rates of $100.0 \%$ compared with $43.3 \%$ in the nonpseudophlebitic group $(P<.0001)$. Dilation of the draining vein was more common in the pseudophlebitic group (72.4\% versus $23.8 \%, P<.0001)$ as was the presence of a venous varix $(63.8 \%$ versus $14.0 \%, P<.0001)$ and draining vein stenosis $(44.8 \%$ versus $15.4 \%$, $P<.0001)$. These data are summarized in Table 2.

\section{MR Imaging Findings}

A total of 191 patients had a preoperative MR imaging (136 in the nonpseudophlebitic group and 55 in the pseudophlebitic group). Cerebral edema was found in $70.9 \%$ of the pseudophlebitic group compared with $2.9 \%$ of the 


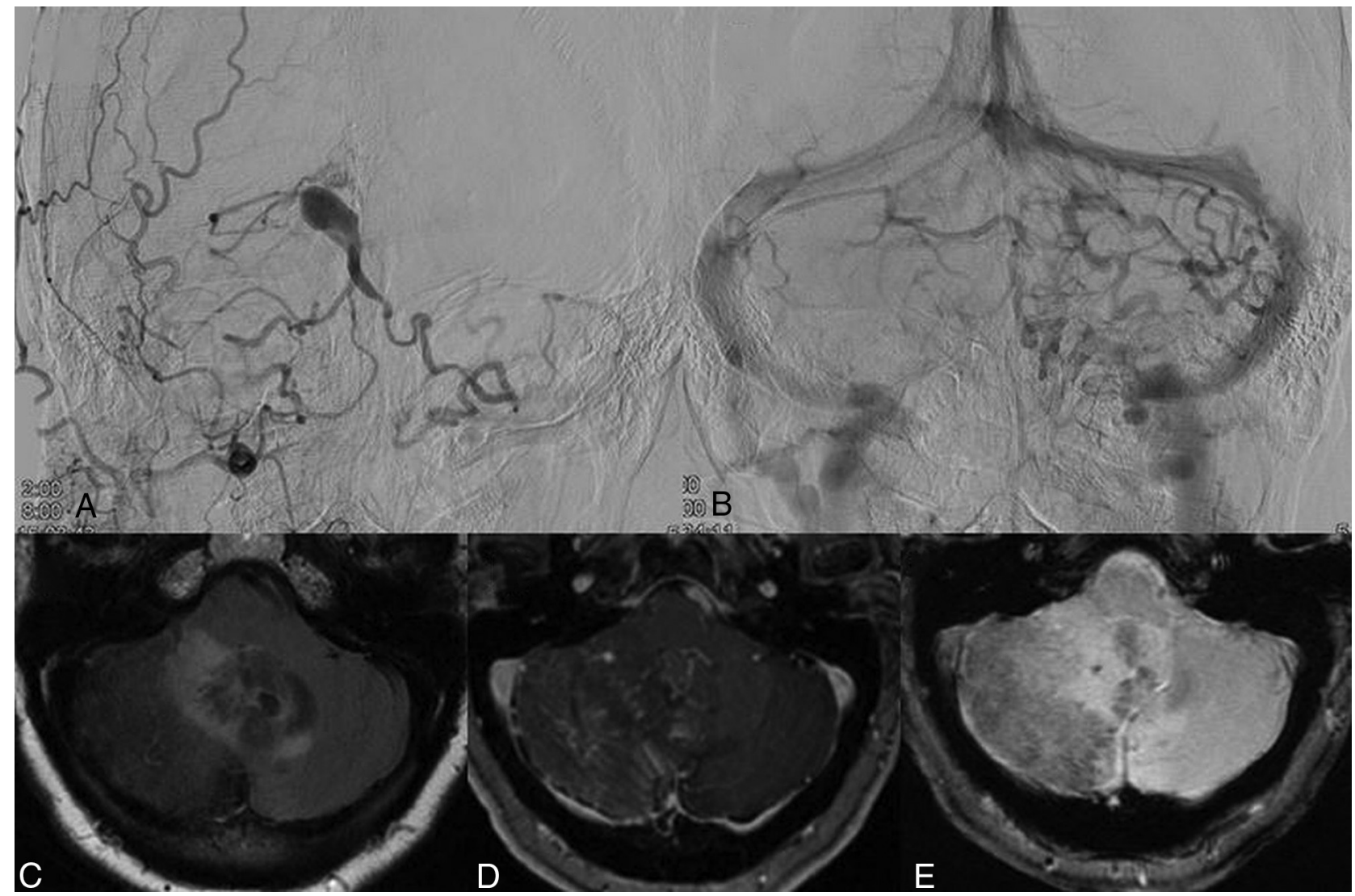

FIG 3. Pseudophlebitic pattern in a patient with a tentorial dural arteriovenous fistula presenting with ataxia. $A$, Right external carotid artery angiogram shows a tentorial dural arteriovenous fistula with reflux into the bilateral cerebellar hemispheres. $B$, Parenchymal venous phase of a left vertebral artery injection shows a pseudophlebitic pattern of the cerebellar parenchymal veins. C, FLAIR MR imaging shows edema in the bilateral cerebellar hemispheres and vermis. D, Postcontrast MR imaging shows dilated transmedullary veins in the right cerebellar hemisphere and vermis. E, Gradient recalled-echo MR imaging shows chronic hemosiderin deposition in the right cerebellar hemisphere.

nonpseudophlebitic group $(P<.0001)$. Chronic hemosiderin deposition and microhemorrhage were found in $17.3 \%$ of the pseudophlebitic group compared with $2.2 \%$ of the nonpseudophlebitic group $(P=.0002)$. Dilated transmedullary veins on SWI or contrast-enhanced T1-weighted images were found in $47.1 \%$ of the pseudophlebitic group compared with $0.0 \%$ of the nonpseudophlebitic group $(P<.0001)$. These data are summarized in Table 3.

\section{Impact of Pseudophlebitic Pattern on Malignant Fistulas}

To determine whether the pseudophlebitic pattern was independently associated with a higher rate of neurologic symptoms and MR imaging findings, we performed a subgroup analysis of patients with malignant fistulas (ie, Borden II/III or cortical venous reflux for cavernous sinus fistulas). A total of 110 patients had malignant fistulas (53 nonpseudophlebitic and 57 pseudophlebitic). There was no difference in hemorrhage at presentation between the 2 groups ( $22.6 \%$ versus $22.8 \%, P=.99$ ). Patients with a pseudophlebitic pattern did have higher rates of nonhemorrhagic neurologic deficits (24.1\% versus $9.4 \%, P=.03$ ). Regarding imaging findings, among patients with malignant fistulas, cerebral edema was more prevalent in patients with a pseudophlebitic pattern $(70.9 \%$ versus $7.6 \%, P<.0001)$ as was chronic hemosiderin deposition $(17.3 \%$ versus $3.9 \%, P=.03)$ and dilated transmedullary veins ( $47.1 \%$ versus $0.0 \%, P<.0001)$.

\section{DISCUSSION}

Our study examining the association between the pseudophlebitic pattern on global angiography and clinical symptoms and imaging findings demonstrated a number of interesting findings. First, the presence of a pseudophlebitic pattern was associated with a significantly higher rate of hemorrhage and other nonhemorrhagic neurologic deficits such as cognitive changes, gait dysfunction, and seizure. Regarding fistula classification, we found that the locations most commonly associated with the presence of a pseudophlebitic pattern were tentorial fistulas $(>50 \%)$ and superior sagittal sinus fistulas (nearly 10\%). As expected, a pseudophlebitic pattern was seen exclusively in malignant fistulas as defined by the Borden and Cognard classification scales. A number of crosssectional MR imaging findings were found to be independently associated with the presence of a pseudophlebitic pattern, including cerebral edema, chronic hemosiderin deposition, and dilated transmedullary veins. These findings are important because they highlight the pseudophlebitic pattern being a reliable prognostic marker for a symptomatic and malignant fistula.

The pseudophlebitic pattern was first described in the setting of cranial dural arteriovenous fistulas by Willinsky et al. ${ }^{4}$ In their series of 122 patients, a pseudophlebitic pattern was seen in 51 patients, (42\%) most of whom presented with hemorrhage or nonhemorrhagic neurologic deficits. Similar to our study, Willinsky et al found 


\begin{tabular}{|c|c|c|c|c|}
\hline & Overall & Nonpseudophlebitic & Pseudophlebitic & $P$ \\
\hline \multicolumn{5}{|l|}{ Fistula location } \\
\hline Transverse sigmoid & $63(31.3)$ & $58(40.6)$ & $5(8.6)$ & $<.0001$ \\
\hline Cavernous sinus & $27(13.4)$ & $23(16.1)$ & $4(6.9)$ & \\
\hline Tentorial & $54(26.9)$ & $24(16.8)$ & $30(51.7)$ & \\
\hline Foramen magnum & $7(3.5)$ & $4(2.8)$ & $3(5.2)$ & \\
\hline Superior sagittal sinus & $6(3.0)$ & $1(0.7)$ & $5(8.6)$ & \\
\hline Anterior cranial fossa & $5(2.5)$ & $5(3.5)$ & $0(0.0)$ & \\
\hline Extensive & $1(0.5)$ & $1(0.7)$ & $0(0.0)$ & \\
\hline Multiple & $9(4.5)$ & $4(2.8)$ & $5(8.6)$ & \\
\hline Hypoglossal/jugular foramen & $16(8.0)$ & $13(9.1)$ & $3(5.2)$ & \\
\hline Convexity & $7(3.5)$ & $5(3.5)$ & $2(3.5)$ & \\
\hline Middle cranial fossa & $2(1.0)$ & $1(0.7)$ & $1(1.7)$ & \\
\hline Other & $4(2.0)$ & $4(2.8)$ & $0(0.0)$ & \\
\hline \multicolumn{5}{|l|}{ Tentorial location } \\
\hline Superior petrosal sinus & $8(14.6)$ & $3(12.5)$ & $5(16.1)$ & .49 \\
\hline Straight sinus & $1(1.8)$ & $0(0.0)$ & $1(3.2)$ & \\
\hline Galenic & $11(20.0)$ & $4(16.7)$ & $7(22.6)$ & \\
\hline Tentorial sinus & $25(45.5)$ & $14(58.3)$ & $11(35.5)$ & \\
\hline Torcular & $10(18.2)$ & $3(12.5)$ & $7(22.6)$ & \\
\hline \multicolumn{5}{|c|}{ Borden classification (non-cavernous sinus) } \\
\hline I & $68(33.8)$ & $68(56.7)$ & $0(0.0)$ & $<.0001$ \\
\hline II & 27 & $13(10.8)$ & $14(25.9)$ & \\
\hline III & 79 & $39(32.5)$ & 40 (74.1) & \\
\hline \multicolumn{5}{|c|}{ Cognard classification (non-cavernous sinus) } \\
\hline I & 52 & $52(43.3)$ & $0(0.0)$ & \\
\hline Ila & 16 & $16(13.3)$ & $0(0.0)$ & \\
\hline $\mathrm{Ilb}$ & 6 & $5(4.2)$ & $1(1.9)$ & \\
\hline $\mathrm{Ila}+\mathrm{b}$ & 22 & $7(5.8)$ & $15(27.8)$ & $<.0001$ \\
\hline III & 22 & $17(14.2)$ & $5(9.3)$ & \\
\hline IV & 52 & $22(18.3)$ & $30(55.6)$ & \\
\hline V & 4 & $1(0.8)$ & $3(5.6)$ & \\
\hline Venous dilation & $76(37.8)$ & $34(23.8)$ & $42(72.4)$ & $<.0001$ \\
\hline Venous varix & $57(28.4)$ & $20(14.0)$ & $37(63.8)$ & $<.0001$ \\
\hline Draining vein stenosis & $48(23.9)$ & $22(15.4)$ & $26(44.8)$ & $<.0001$ \\
\hline
\end{tabular}

Table 3: Cross-sectional imaging findings

\begin{tabular}{|c|c|c|c|c|}
\hline & Overall & Nonpseudophlebitic & Pseudophlebitic & $P$ \\
\hline Cerebral edema & $43(22.5)$ & $4(2.9)$ & 39 (70.9) & $<.0001$ \\
\hline Chronic hemosiderin deposition/microhemorrhage & $12(6.4)$ & $3(2.2)$ & $9(17.3)$ & .0002 \\
\hline Venous infarct & $2(1.1)$ & $1(0.7)$ & $1(1.9)$ & .49 \\
\hline Dilated transmedullary veins on SWI or CE TI & $24(12.9)$ & $0(0.0)$ & $24(47.1)$ & $<.0001$ \\
\hline Sinus thrombosis & $14(7.4)$ & $8(5.9)$ & $6(11.1)$ & .21 \\
\hline Cortical venous reflux on 3D-TOF & $68(38.9)$ & $32(24.6)$ & $46(80.0)$ & $<.0001$ \\
\hline
\end{tabular}

Note:-CE indicates contrast-enhanced.

that most of the lesions associated with a pseudophlebitic pattern were associated with tentorial or superior sagittal sinus fistulas. Fifty-two patients in their series also had brain MRIs, of whom $20 \%$ had cerebral edema, and $90 \%$ of those with cerebral edema had a pseudophlebitic pattern. Our study adds to this study in that we report more extensively on the MR imaging findings of 191 patients, $95 \%$ of the patients included in this study.

Our study highlights additional imaging findings that are unique to those with a pseudophlebitic pattern. First, we found that the prevalence of cerebral edema among patients with a pseudophlebitic pattern was $71 \%$, significantly higher than the $20 \%$ reported in previous studies. ${ }^{4}$ This prevalence is likely a function of improved imaging techniques (ie, increased use of
FLAIR, routine 1.5T and 3T MR imaging, and so forth). Cerebral edema in these patients is likely a reflection of diffuse venous congestion (congestive edema) resulting in fluid retention in the interstitial tissues. We found that $17 \%$ of patients had chronic hemosiderin deposition or microhemorrhage on $\mathrm{T} 2{ }^{\star}$-weighted imaging, which is likely a function of tiny perivenular microhemorrhages in the setting of severe venous hypertension. Dilated transmedullary veins on SWI or contrast-enhanced T1 imaging were found in nearly $50 \%$ of patients with a pseudophlebitic pattern, a unique association, likely a function of rerouting of normal cerebral venous drainage via transmedullary venous pathways. ${ }^{7}$ We did not find any association between venous sinus narrowing or thrombosis and the presence of a pseudophlebitic 
pattern. This finding is likely because in most cases of dural venous sinus thrombosis-associated dAVFs, the lesions are located in the transverse and sigmoid sinuses and there are multiple venous egress pathways available for the draining cortical veins. Tentorial and ethmoidal dAVFs are less likely to be associated with venous sinus thrombosis or stenosis.

The pseudophlebitic pattern was not independently associated with hemorrhage but was independently associated with the presence of nonhemorrhagic neurologic deficits. Previous studies have suggested that the pseudophlebitic pattern is a risk factor for hemorrhage, but they did not stratify lesions by the presence or absence of retrograde leptomeningeal venous drainage. ${ }^{4}$ When we examined patients who had retrograde leptomeningeal venous drainage, we found that the rate of hemorrhage was similar between those who did and did not have a pseudophlebitic pattern. This finding suggests that the primary driver of hemorrhage is the venous pathology along the draining vein rather than the venous congestion in the affected territory. However, the fact that nonhemorrhagic neurologic deficits such as seizure and cognitive decline are more common among those with a pseudophlebitic pattern suggests that the chronic venous congestion resulting from the normal brain not being able to properly drain in the setting of leptomeningeal venous drainage is the primary driver of these particular symptoms. ${ }^{3,8-10}$ This feature has been suggested by prior reports on the characteristics of dAVFs associated with cognitive decline, which have suggested that nearly all of these patients have evidence of medullary venous hypertension with reflux or congestion of the transmedullary veins on conventional imaging and angiography. ${ }^{11}$ Given the strong association between the pseudophlebitic pattern and nonhemorrhagic neurologic deficits in our study, we think that the presence of this pattern should encourage the neurointerventionalist or neurosurgeon to be more aggressive in treating and curing the fistula to prevent the downstream effects of chronic venous congestion.

One additional interesting finding from our study was the location of most lesions associated with the pseudophlebitic pattern. Most of these lesions were more or less midline in location (ie, tentorial or superior sagittal sinus). Tentorial dAVFs involving the midline tentorial sinuses, the torcula, or the vein of Galen cause significant venous hypertension in the vein of Galenstraight sinus-torcula draining pathway. Thus, in such cases, the primary means by which the deep venous system drains into the dural venous sinuses is under high pressure, resulting in high pressure in the parenchymal veins draining into the deep venous system and a pseudophlebitic congestive pattern. The same is true for superior sagittal sinus fistulas because the cortical veins experience markedly elevated venous pressure resulting in congestion of the parenchymal veins and an associated pseudophlebitic pattern. This feature would be less common in laterally located lesions (ie, transverse sigmoid junction fistulas) because there may be more avenues for venous egress for the parenchymal veins.

Regarding sex, we found that male sex was associated with a pseudophlebitic pattern on angiography. A number of large clinical case series have found that male sex is associated with a more aggressive angioarchitecture of cranial dural arteriovenous fistulas; however, the reason for this association is unclear. ${ }^{4,12,13}$ In their review of $>300$ cranial and spinal arteriovenous fistulas, Geibprassert et al noticed that fistulas associated with more benign locations and angioarchitecture had a female predominance while those in locations associated with more malignant angioarchitecture were much more common in men. ${ }^{13}$ These findings point to a difference in the etiology of cranial dural arteriovenous fistulas by location. We hypothesize that women are more likely to have the more benign fistulas in the cavernous sinus and transverse sigmoid region due to their propensity to have dural venous sinus thromboses, while men are more likely to have aggressive tentorial or anterior ethmoidal fistulas due to higher rates of trauma.

\section{Limitations}

Our study has limitations. This patient population is from a 12year time span. During the time period, there have been significant advancements in neuroimaging with increased use of susceptibility-weighted imaging over gradient recalled-echo and higher-resolution FLAIR sequences, which could increase the sensitivity for detecting microhemorrhages and edema, respectively. In addition, imaging was performed across a variety of scanners with $1.5 \mathrm{~T}$ and $3 \mathrm{~T}$ field strengths, so there was not $100 \%$ uniformity in the imaging protocols. All cross-sectional imaging interpretation was performed by a single neuroradiologist. Last, we did not include multiphase imaging in our study (ie, Time Resolved Imaging of Contrast Kinetics). It is possible that this could have a role in understanding the presence of a pseudophlebitic pattern before conventional angiography.

\section{CONCLUSIONS}

Our study of 201 cranial dural arteriovenous fistulas, 191 of which had MR imaging, found that the pseudophlebitic pattern was associated with more aggressive dural arteriovenous fistulas. When we examined patients with retrograde leptomeningeal venous drainage, the pseudophlebitic pattern was independently associated with nonhemorrhagic neurologic deficits, but not hemorrhage. MR imaging findings most commonly associated with the pseudophlebitic pattern were cerebral edema, dilated transmedullary veins on postcontrast or $\mathrm{T} 2^{\star}$-weighted imaging, and chronic hemosiderin deposition/microhemorrhage. These findings can help in the risk stratification of dural arteriovenous fistulas.

Disclosures: Giuseppe Lanzino-UNRELATED: Board Membership: Superior Medical Editing, Nested Knowledge.

\section{REFERENCES}

1. Reynolds MR, Lanzino G, Zipfel GJ. Intracranial dural arteriovenous fistulae. Stroke 2017;48:1424-31 CrossRef Medline

2. Biondi A, Casasco A, Houdart E, et al. Evolution of angiographic signs of venous hypertension and clinical signs of intracranial hypertension in intracranial dural arteriovenous fistulas [in French]. J Neuroradiol 1999;26:49-58 Medline

3. Cognard C, Casasco A, Toevi M, et al. Dural arteriovenous fistulas as a cause of intracranial hypertension due to impairment of cranial venous outflow. J Neurol Neurosurg Psychiatry 1998;65:308-16 CrossRef Medline

4. Willinsky R, Goyal M, terBrugge $\mathrm{K}$, et al. Tortuous, engorged pial veins in intracranial dural arteriovenous fistulas: correlations with 
presentation, location, and MR findings in 122 patients. AJNR Am J Neuroradiol 1999;20:1031-36 Medline

5. Osada $\mathrm{T}$, Krings $\mathrm{T}$. Intracranial dural arteriovenous fistulas with pial arterial supply. Neurosurgery 2019;84:104-15 CrossRef Medline

6. Lawton MT, Sanchez-Mejia RO, Pham D, et al. Tentorial dural arteriovenous fistulae: operative strategies and microsurgical results for six types. Neurosurgery 2008;62:110-24; discussion 24-25 CrossRef Medline

7. Nakagawa I, Taoka T, Wada T, et al. The use of susceptibility-weighted imaging as an indicator of retrograde leptomeningeal venous drainage and venous congestion with dural arteriovenous fistula: diagnosis and follow-up after treatment. Neurosurgery 2013;72:47-54; discussion 55 CrossRef Medline

8. Iwama T, Hashimoto N, Takagi $\mathrm{Y}$, et al. Hemodynamic and metabolic disturbances in patients with intracranial dural arteriovenous fistulas: positron emission tomography evaluation before and after treatment. J Neurosurg 1997;86:806-11 CrossRef Medline

9. Kanemaru K, Kinouchi H, Yoshioka H, et al. Cerebral hemodynamic disturbance in dural arteriovenous fistula with retrograde leptomeningeal venous drainage: a prospective study using (123) I-iodoamphetamine single photon emission computed tomography. J Neurosurg 2015;123:110-17 CrossRef Medline

10. Kwon BJ, Han MH, Kang HS, et al. MR imaging findings of intracranial dural arteriovenous fistulas: relations with venous drainage patterns. AJNR Am J Neuroradiol 2005;26:2500-07 Medline

11. Brito A, Tsang AC, Hilditch C, et al. Epidemiology of dural arteriovenous fistula in Japan: analysis of Japanese Registry of Neuroendovascular Therapy (JR-NET2). Neurol Med Chir (Tokyo) 2019;121:e543-53 Medline

12. Brito A, Tsang AC, Hilditch $C$, et al. Intracranial dural arteriovenous fistula as a reversible cause of dementia: case series and literature review. World Neurosurg 2014;54(Suppl 2):63-71 CrossRef Medline

13. Geibprasert S, Pereira V, Krings T, et al. Dural arteriovenous shunts: a new classification of craniospinal epidural venous anatomical bases and clinical correlations. Stroke 2008;10:2783-94 CrossRef Medline 